\title{
Spectrum Sharing with Smooth Supermodular Game in Cognitive Radio Networks
}

\author{
Huili Cheng, Qinghai Yang, Fenglin Fu \\ State Key Lab. of ISN, School of Telecomm. \\ Engineering, Xidian University, No.2 Taibainan-lu, Xi'an, \\ 710071, Shaanxi,China. Email: qhyang@xidian.edu.cn
}

\author{
Kyung Sup Kwak \\ Graduate School of IT and Telecomm., \\ Inha University, \#253 Yonghyun-dong, Nam-gu, \\ Incheon, 402-751, Korea. Email: kskwak@inha.ac.kr
}

\begin{abstract}
In this paper, a supermodular game theoretic approach is investigated for spectrum sharing in cognitive radio networks. We consider a Bertrand competition model, in which primary service providers compete to sell their spare spectrum and then to maximize their individual profits. We demonstrate that the Bertrand competition is a smooth supermodular game, and a round-robin optimization algorithm is developed to obtain the optimal price solutions. Simulation results verify that the algorithm approximately converges to an equilibrium point, and the influence of the exogenous variable on the equilibrium point is analyzed.
\end{abstract}

Index Terms-spectrum sharing, smooth supermodular game, Bertrand competition, exogenous variable.

\section{INTRODUCTION}

Wireless communications technology which is based on the transmission of radio waves has become a key element in modern life. Most of the available radio spectrum has already been allocated to existing wireless systems. However, many frequency bands are only partly used or largely unoccupied [1-2]. Cognitive radio is a new paradigm of designing wireless communication systems which aims to enhance the utilization of the radio frequency spectrum. Implementation of cognitive radio will be based on dynamic spectrum access by the unlicensed users [3-4]. Frequency bands which are not occupied can be sold to unlicensed users by licensed users, and then the market of the spectrum trading appears. In spectrum trading, the key element is the price of spectral resources, which should be properly decided based on the objectives of both licensed and unlicensed users.

In a cognitive radio network, the problem of pricing is somewhat different from that in a traditional wireless network due to the spectrum sharing and the adaptability of the licensed and unlicensed users. A licensed user can charge an unlicensed user for spectrum access. This price can be dynamically adjusted in proportional to the availability of spectrum opportunity, which is a function of the traffic load in the licensed network and the demand from the unlicensed

\footnotetext{
*This research was supported by the RCUK for the UK-China Science Bridges Project: R\&D on (B) $4 \mathrm{G}$ wireless mobile communications, the MKE, Korea, under the ITRC program supervised by the NIPA (NIPA-2011-C10901121-0001) and the Fundamental Research Funds for the Central Universities (JY10000901002, JY10000901020), NSF China (60832001, 61001127), the 111 Project (B08038), China.
}

users. This demand depends on the current number of ongoing sessions and applications used by the unlicensed users.

In this paper, we regard the spectrum trading as a Bertrand game [5-6], in which the players are the primary service providers (PSP) and the strategy is the price per unit spectrum. PSPs compete with each other to sell spectrum to secondary service providers (SSP) whilst aiming to maximize their individual profits [7-8].

The main contributions of this paper are as follows:

- Bertrand competition model is presented for spectrum trading in cognitive radio networks, which is proved as a smooth supermodular game [9].

- Simulations based on round-robin optimization in [10] are provided to approximate an equilibrium point, and solutions are obtained for this model.

- Extensive analysis of the exogenous variable's influence on the equilibrium is provided.

The rest of this paper is organized as follows. In Sec. II, we present the basic knowledge of the supermodular game, smooth supermodular game and Bertrand competition theories. The system model is introduced in Sec. III. The solutions of the spectrum price problem is presented in Sec. IV. Simulation results are provided in Sec. V, and Sec. VI concludes this paper.

\section{Preliminary}

\section{A. Supermodular Game}

Suppose that $X$ is a partially ordered set and $X^{\prime}$ is a subset of $X$. If $x^{\prime}$ is in $X$ and $x \prec x^{\prime}\left(x^{\prime} \prec x\right)$ for each $x$ in $X^{\prime}$, then $x^{\prime}$ is an upper (lower) bound for $X^{\prime}$. If $x^{\prime}$ in $X^{\prime}$ is an upper (lower) bound for $X^{\prime}$, then $x^{\prime}$ is the greatest (least) element of $X^{\prime}$. If the set of upper (lower) bounds of $X^{\prime}$ has a least (greatest) element, then this least upper bound (greatest lower bound) of $X^{\prime}$ is the supremum (infimum) of $X^{\prime}$. If two elements, $x$ and $x^{\prime}$, of a partially ordered set $X$ have a supremum (infimum) in $X$, it is their joint (meet) and is denoted as $x \vee x^{\prime}$. A partially ordered set that contains the joint and the meet of each pair of its elements is a lattice. If $X^{\prime}$ is a subset of a lattice $X$ and $X^{\prime}$ contains the joint and meet (with respect to $X$ ) of each pair of elements of $X^{\prime}$, then $X^{\prime}$ is a sublattice of $X$.

Suppose that $f(x)$ is a real-valued function on a lattice $X$. If $f(x)+f\left(x^{\prime}\right) \leq f\left(x \vee x^{\prime}\right)+f\left(x \wedge x^{\prime}\right)$ for all $x$ and $x^{\prime}$ in $X$, 
then, $f(x)$ is a supermodular function on $X$. Suppose that $X$ and $T$ are partially ordered sets and $f(x, t)$ is a real-valued function on a subset $S$ of $X \times T$ (direct product). For $t$ in $T$, let $S_{t}$ denote the section of $S$ at . If $f\left(x, t^{\prime \prime}\right)-f\left(x, t^{\prime}\right)$ is increasing in $x$ on $S_{t^{\prime \prime}} \cap S_{t^{\prime}}$ for all $t^{\prime} \prec t^{\prime \prime}$ in $T$, then $f(x, t)$ has increasing difference in $(x, t)$ on $S$.

A noncooperative game $\left(N, S, f_{i}: i \in\{1, \cdots, N\}\right)$, in which the strategy of player $i$ is an $m_{i}$ vector $x_{i}$, is a supermodular game if the set $S$ of feasible joint strategies is a sublattice of $R^{m}$ (or of $\times_{i \in\{1, \cdots, N\}} R^{m_{i}}, m=\Sigma_{i=1}^{N} m_{i}$ ), the payoff function $f_{i}\left(y_{i}, x_{-i}\right)$ is supermodular in $y_{i}$ on $S_{i}$ for each $x_{-i}$ in $S_{-i}$ and each player $i$, and $f_{i}\left(y_{i}, x_{-i}\right)$ has increasing difference in $\left(y_{i}, x_{-i}\right)$ on $S_{i} \times S_{-i}$ for each $i$.

Theorem 1 [10]: If the game is supermodular, there exists a largest and a smallest Nash equilibrium in pure strategies.

\section{B. Smooth Supermodular Game}

Since the strategy space of the game in this paper is continuous, for vectors $x=\left(x_{1}, \cdots, x_{n}\right)$ and $y=\left(y_{1}, \cdots, y_{n}\right)$ in the $n$ dimension Euclid space, $x \geq y$ is equivalent to $x_{i} \geq y_{i}(i=1, \cdots, n)$. Furthermore, certain theorems are specializations of primitive theorems due to the smoothness of the profit function.

Lemma 1[10]: The necessary and sufficient condition for a function $f$ having increasing differences on $R^{n}$ is that the function $f$ is supermodular.

Lemma 2[10]: If a function $f: R^{n} \rightarrow R$ is twice continuously differentiable in an open interval including a interval $I=[\underline{x}, \bar{x}]$ in $R^{n}$, then the necessary and sufficient condition of the function $f$ having the supermodularity is $\frac{\partial^{2} f(x, y)}{\partial x_{i} \partial x_{j}} \geq 0, \forall i \neq j$.

Accordingly, the request of supermodularity is easily satisfied for an general twice continuously differentiable function, since it just needs the nonnegativity condition of the cross partial derivative. The definition of the smooth supermodular game is provided below.

Suppose that there are $N$ players in a game, subscripted by $m$ and $n$, and player $n$ has a strategy consisting of $k_{n}$ elements, subscripted by $i$ and $j$, so its strategy is the vector $\mathbf{x}_{\mathbf{n}}=\left(x_{n 1}, \cdots, x_{n k}\right)$. Define the strategy set as $S_{n}$ and the payoff function as $\pi_{n}\left(x_{n}, x_{-n} ; \tau\right)$, where $\tau$ is a fixed parameter. We say that a game is a smooth supermodular game if the following four conditions are satisfied for every player.

(A) The strategy set is an interval in $R^{k_{n}}: S_{n}=\left[x_{n}, \overline{x_{n}}\right]$. $S_{n}$

(B) $\pi_{n}\left(x_{n}, x_{-n} ; \tau\right)$ is twice continuously differentiable on

(C) (Supermodularity) Increasing one component of player $n$ 's strategy does not decrease the net marginal benefit of any other component for all $n$, and all $i$ and $j$ such that $1 \leq i<$ $j \leq k_{n}$,

$$
\frac{\partial^{2} \pi_{n}}{\partial x_{n i} \partial x_{n j}} \geq 0 .
$$

(D) (Increasing differences in one's own and other strategies) Increasing one component of $n$ 's strategy does not decrease the net marginal benefit of increasing any component of player $m$ 's strategy: for all $m \neq n$, and all $i$ and $j$ such that $1 \leq i \leq k_{n}, 1 \leq j \leq k_{m}$

$$
\frac{\partial^{2} \pi_{n}}{\partial x_{n i} \partial x_{m j}} \geq 0 .
$$

In addition, we will be able to talk about the comparative static of smooth supermodular games if a fifth condition is satisfied, the increasing difference condition, (E).

(E) (Increasing difference in one's own strategies and parameters) Increasing parameter $\tau$ does not decrease the net marginal benefit to player $n$ of any component of its own strategy: for all $n$, and $i$ such that $1 \leq i \leq k_{n}$,

$$
\frac{\partial^{2} \pi_{n}}{\partial x_{n i} \partial \tau} \geq 0 .
$$

Theorem 2: If the game is supermodular and the assumption of (E) is satisfied, then the largest and the smallest equilibrium are nondecreasing functions of the parameter $\tau$ [9].

\section{Bertrand Competition}

Bertrand competition is a model of competition used in economics, which describes interactions among firms (sellers) that set prices and their customers (buyers) that choose quantities at that price. It follows the assumptions:

- There are at least two firms producing homogenous (undifferentiated) products;

- Firms do not cooperate;

- Firms compete by setting prices simultaneously;

- Consumers buy everything from a firm with a lower price. If all firms charge the same price, consumers randomly select among them.

\section{SYSTEM MODEL}

We consider a cognitive radio system with $N$ PSPs. PSP $i$ ( $i \in\{1, \cdots, N\}$ ) owns a size of $B_{i}$ frequency spectrum and it serves $K_{i}$ primary users (PU) as shown in Fig.1. When a PSP is not fully utilizing its spectrum, it can sell portions of the available spectrum to SSPs, whose users are called secondary users (SU). In this way, a spectrum trading market is established. The price per spectrum unit of $B_{i}$ is denoted as $p_{i}$.

Since there are more than two PSPs, who do not cooperate with each other and will announce prices simultaneously, we shall adopt the Bertrand competition model to set the price. In fact, the Bertrand competition can be viewed as a noncooperative game. We assume that each PSP is aware of the existence of other PSPs and all of them compete with each other to maximize their individual profits. The PSPs are viewed as players and the price per spectrum unit of $B_{i}$ is the strategy of PSP $i$. Each PSP gains the profit via selling spectrum to the SSP.

In Fig.1, the SSP is a fictional service provider (a representative consumer [11]), whose utility maximization problem generates the aggregate demand functions when facing aggregate resource constraints. The assumption does not rule out SSP heterogeneity, but only requires that the potential sources 


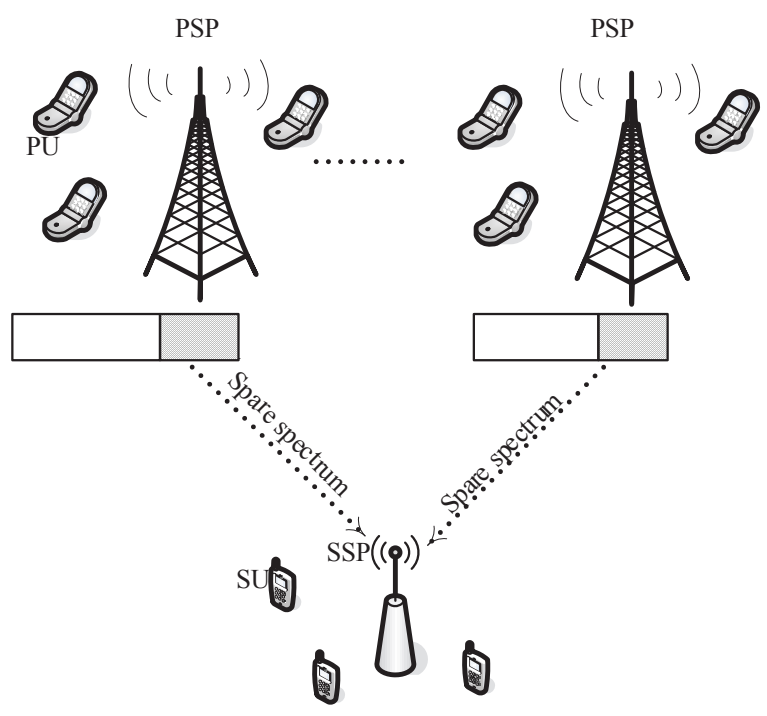

Fig. 1. Spectrum sharing model

of the heterogeneity have sufficient structure to ensure that the cluster of all SSPs behaves as if it were a single provider.

\section{Solution of Spectrum Price Problem}

\section{A. Demand Function}

Assuming a cluster of SSPs behaves as if it were a single one, the spectrum demand of such a virtual provider can be expressed directly as follows [12],

$$
D_{i}(\mathbf{p}, d)=a_{i}-b_{i} p_{i}+d \sum_{j \neq i} p_{j}
$$

where $p_{i}$ is the price offered by PSP $i, \mathbf{p}$ is the price vector with component $p_{i}, a_{i}$ denotes the market capacity of the spectrum $B_{i}$, and $d$ is the substitutability coefficient $\left(0 \leq d \leq b_{i}\right)$. When $d$ is the upper bound, it implies that the two spectrums can be completely substituted. Moreover, the spectrums become independent when $d$ equals to zero.

\section{B. Profit Function}

This noncooperative game, a single decision variable (price) competition, is a Bertrand competition. The profit function of PSP is developed as follows:

$$
u_{i}(\mathbf{p}, d)=\left(p_{i}-c_{i}\right) D_{i}(\mathbf{p}, d)-\nu_{i}\left(Q_{i}-\frac{B_{i}-D_{i}(\mathbf{p}, d)}{K_{i}}\right),
$$

where $\nu_{i}$ is the coefficient of the penalty term, $Q_{i}$ denotes the bandwidth requirement per $\mathrm{PU}$ and $c_{i}$ denotes the cost of per spectrum unit of $D_{i}(\mathbf{p}, d)$. PSP $i(i \in\{1, \cdots, N\})$ owns a size of $B_{i}$ frequency spectrum and it serves $K_{i}$ primary users.

In this case, $p_{i} D_{i}(\mathbf{p}, d)$ denotes the revenue gained from sharing spectrum with $\mathrm{SSP}$, and $c_{i} D_{i}(\mathbf{p}, d)$ denotes the cost of PSP $i$ for owning the spectrum $D_{i}(\mathbf{p}, d)$. Here, the cost involved is a function of QoS performance degradation [13] of ongoing PUs due to sharing spectrum with SSP.
Proposition 1:The game with utility function (5) is a smooth supermodular game.

Proof: We suppose the price is restricted to $\left[c_{i}, \bar{p}\right]$ for player $i$, where $\bar{p}$ can be chosen to yield zero quantity demand, so (A) is satisfied. $u_{i}(\mathbf{p}, d)$ is twice continuously differentiable on $\left[c_{i}, \bar{p}\right]$, so (B) is satisfied. As explained above, this is a single decision variable (price) competition, that is, a player's strategy has just one component, $p_{i}$, so (C) is satisfied.

The first-order derivative of the profit function is

$$
\begin{gathered}
\frac{\partial u_{i}(\mathbf{p}, d)}{\partial p_{i}}=D_{i}(\mathbf{p}, d)+\left(p_{i}-c_{i}\right) \frac{\partial D_{i}(\mathbf{p}, d)}{\partial p_{i}} \\
-2 \frac{\nu_{i}}{K_{i}}\left(Q_{i}-\frac{B_{i}-D_{i}(\mathbf{p}, d)}{K_{i}}\right) \frac{\partial D_{i}(\mathbf{p}, d)}{\partial p_{i}} .
\end{gathered}
$$

The following inequality is true,

$$
\frac{\partial^{2} u_{i}(\mathbf{p}, d)}{\partial p_{i} \partial p_{j}}=d+\frac{2 b_{i} d \nu_{i}}{K_{i}^{2}} \geq 0,
$$

so (D) is satisfied. And it is also true that

$$
\frac{\partial^{2} u_{i}(\mathbf{p}, d)}{\partial p_{i} \partial d}=\left(1+\frac{2 b_{i} \nu_{i}}{K_{i}}\right) \Sigma_{j \neq i} p_{j} \geq 0,
$$

so (E) is satisfied for $d$. Then the game is a smooth supermodular game.

By Theorem 1 and 2, it yields the following results for our differentiated Bertrand game:

- There exists a largest and a smallest Nash equilibrium in pure strategies (Theorem 1);

- The largest and the smallest equilibrium prices for PSP $i$ are nondecreasing functions of the parameter $d$ (Theorem 2).

The best response is given by

$$
\mathbf{U}_{\mathbf{i}}\left(p_{-i}\right)=\max _{p_{i} \in \mathbf{P}_{\mathbf{i}}\left(p_{-i}\right)} u_{i}\left(p_{i}, p_{-i} ; d\right) .
$$

For any joint strategy $\mathbf{p} \in \mathcal{P}$ ( $\mathcal{P}$ is the strategy space) and any player $i, p_{-i}$ denotes the vector of strategies of all players in $1, \cdots, N$ except player $i$. And $\left(p_{i}, p_{-i}\right)$ denotes the joint strategy vector with $p_{i}$ being changeable and the other components of $\mathbf{p}$ being constant. The set of feasible strategies for player $i$ given strategies $p_{-i}$ for the other players is denoted as $\mathbf{p}_{\mathbf{i}}\left(p_{-i}\right)=p_{i}:\left(p_{i}, p_{-i}\right) \in P$. The substitutability coefficient $d$ is an exogenous variable used in comparative static analysis.

\section{Algorithm}

Based on round-robin optimization, we shall develop the algorithm to reach the optimal solutions. Given a noncooperative game $\left(N, P, u_{i}: i \in\{1, \cdots, N\}\right)$, a sequence of feasible joint strategies is obtained as:

Step1: Set $d=0$. If $\mathcal{P}$ has a least element $\inf (\mathcal{P})$, set $\mathbf{p}^{\mathbf{0 , 0}}=\inf (\mathcal{P})$. Otherwise, stop.

Step2: Given $\mathbf{p}^{\mathbf{k}, \mathbf{i}}$ in $\mathcal{P}$ for any $k$ and $i$ with $k \geq 1,1 \leq i \leq$ $N$, let $\mathbf{p}^{\mathbf{k}, \mathbf{i}}=\left(p_{i}^{k, i}, p_{-i}^{k, i-1}\right)$, where $p_{i}^{k, i}$ is the least element of the best response (9), if such an element exists. Otherwise, stop. 
Step3: Set $i=i+1$ if $i=N$ so $\mathbf{p}^{\mathbf{k}, \mathbf{N}}$ has been generated for some $k$, set $\mathbf{p}^{\mathbf{k}+\mathbf{1}, \mathbf{0}}=\mathbf{p}^{\mathbf{k}, \mathbf{N}}$, set $k=k+1$, and set $i=0$. If $k \leq N$ go to step 3 and continue, otherwise write $\mathbf{p}^{\mathbf{N}, \mathbf{N}}$ into a matrix $F$.

Step4: Set $d=d+0.1$, if $d \leq b_{i}$, go to step3, otherwise, stop.

\section{Simulation AND Discussion}

We cosider a cognitive radio system with three PSPs and each PSP has $K_{i}=10$ PUs. The total spectrum of PSPs are $200 \mathrm{MHz}, 200 \mathrm{MHz}$ and $300 \mathrm{MHz}$, respectively (i.e., $\mathbf{B}=(200,200,300))$. Suppose the spectrum requirement of PUs from their PSPs are $20 \mathrm{MHz}, 20 \mathrm{MHz}$ and30 MHz, respectively (i.e., $\mathbf{Q}=(20,20,30))$. The spectrum capacity of the market of each PSP is $200 \mathrm{MHz}, 200 \mathrm{MHz}$ and 300 $\mathrm{MHz}$, respectively, $\mathbf{a}=(200,200,300)$. The coefficient of penalty item is $\nu=(1,2,3)$ and the cost of per spectrum unit is $\mathbf{c}=(2,3,4)$. Note that substitutability coefficient $d\left(0 \leq d \leq b_{i}\right)$ is considered as an exogenous variable when we talk about the comparative statics of smooth supermodular games.

\section{A. Existence of Nash Equilibrium}

The algorithm above generates an infinite sequence $\mathbf{p}^{\mathbf{k}, \mathbf{i}}$ that is increasing in $k$ and $i$ for $k \geq 1,1 \leq i \leq N$. Hence, there exists a feasible joint strategy $\mathbf{p}^{\prime}$ in $\mathcal{P}$ such that $\lim _{k \rightarrow \infty} \mathbf{p}^{\mathbf{k}, \mathbf{i}}=\mathbf{p}^{\prime}$. If a feasible joint strategy appears $n$ times successively in the sequence $\mathbf{p}^{\mathbf{k}, \mathbf{i}}$, then it is an equilibrium point. Fig. 2 shows that the prices per spectrum unit for the three PSPs are increasing in iteration and then reach a stable state, the Nash equilibrium point. Here one can't improve its individual profit even if it increases its price. At last, the three PSPs will share their spectrums with the SSP at these prices.

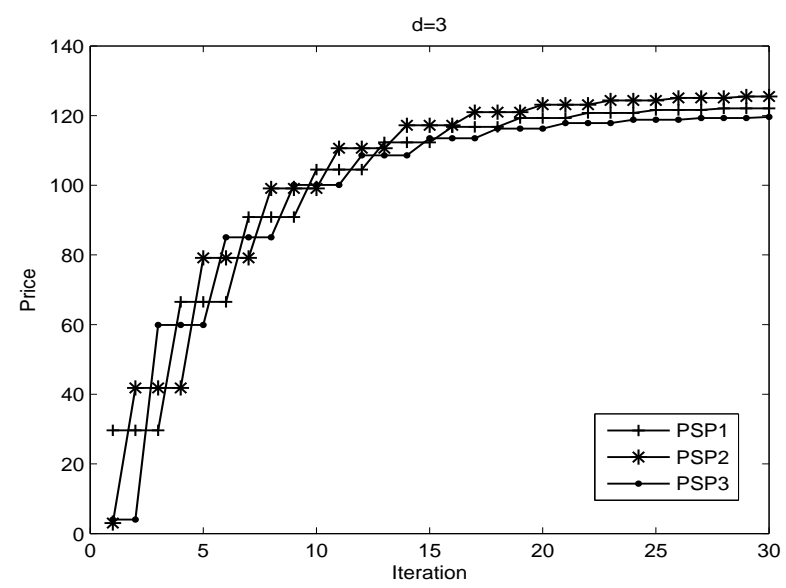

Fig. 2. Price versus iteration for three primary service providers

\section{B. Comparative Static Analysis on d}

Comparative static is the analysis of what happens to endogenous variables in a model when the exogenous variable changes. A small change in the parameter might lead to a

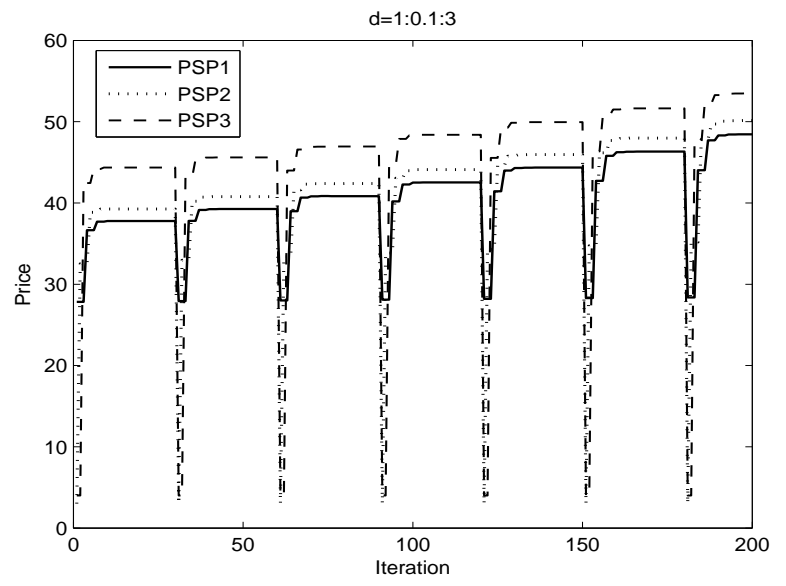

Fig. 3. Price versus $d$ and iteration for three primary service providers

large change in the equilibrium because of feedback among the different players' strategies.

Fig. 3 shows that the system can reach a stable state for different $d$, and the Nash equilibrium point increases versus $d$, where $d=1: 0.1: 3$. As expected, the supermodularity yields comparative statics on $d$, the discrete exogenous variable. However, because it just finds the effect of $d$ on $\mathbf{p}^{\mathbf{k}, \mathbf{i}}$, rather than telling us its exact value, it yields weak comparative static on $d$. The frequency of the SSP switching between different spectrums increases as $d$ increased. This implies that the price in equilibrium point will increase when it is easy to switch.

The scheme proposed in this paper decreases the complexity of calculating equilibrium points and, for the profit function, just requires the nonnegativity condition of the cross partial derivative, regardless of what its second order partial derivative and concavity are, as compared with that of the general game methodology. Moreover, comparative static analysis, (i.e. the change of equilibrium points on the exogenous variable) is made to predict strategies of players when external conditions change.

\section{CONCLUSIONS}

In this paper, we consider the spectrum trading, a Bertrand competition, as a noncooperative game, in which the players are PSPs and the strategy is the price per spectrum unit. PSPs compete with each other to maximize their individual profits. We prove that the Bertrand competition following a supermodular game, and the round-robin optimization algorithm is developed to approximate the Nash equilibrium. We provide comparative static analysis for the supermodular game, in which the price vector in equilibrium point increases as substitutability coefficient increased, i.e. the smaller spectrum difference leads to the higher prices.

\section{REFERENCES}

[1] S. Haykin, "Cognitive radio: brain-empowered wireless communications," Journal on Selected Areas in Communications, vol. 23, no. 2, pp. 201220, 2005. 
[2] I. F. Akyildiz,W.-Y. Lee, M. C.Vuran, and S. Mohanty, "Next generation/dynamic spectrum access/cognitive radio wireless networks: a survey," Computer Networks, vol. 50, no. 13, pp. 2127-2159, 2006.

[3] D. Cabric, S. M. Mishra, D. Willkomm, R. Brodersen, and A. Wolisz, "A cognitive radio approach for usage of virtual unlicensed spectrum," in Proceedings of 14th IST Mobile and Wireless Communications Summit, June 2005.

[4] Ekram Hossain, Dusit Niyato and Zhu Han, Dynamic spectrum access and management in cognitive radio networks . Cambridge University Press, 2009, pp. 39-46.

[5] W. W. Sharkey and D. S. Sibley, "A Bertrand model of pricing and entry," Economics Letters, 41:199-206, 1993.

[6] Yang Xiao-hua, "Bertrand model and the theory of supermodular games,"Chinese joural of management science, vol.17, no.1, pp. 95-100, Feb. 2009.

[7] G. Owen, Game Theory. Academic Press, 2001

[8] D. Fudenberg and J. Tirole, Game Theory. MIT Press, 1991.

[9] Eric Rasmusen, Games and information, fourth edition: an introduction to game theory. Blackwell Publishers, 2005, pp.355-360.

[10] Topkis M D, Supermodularity and complementarity. Princeton: Princeton University Press, 1998.

[11] Francesco Caselli and Jaume Ventura, "A representative consumer theory of distribution," The American economic review, Vol. 90, No. 4, pp. 909926, Sep. 2000.

[12] N. Singh and X.Vives, "Price and quantity competition in a differented duopoly," Rand J.Economics, vol.15, no.4, pp.546-554, 1984.

[13] D. Niyato and E. Hossain, "Market-equilibrium, competitive, and cooperative pricing for spectrumsharing in cognitive radio networks: analysis and comparison," IEEE Transactions on Wireless Communications, vol. 7, no. 11 (Part 1), pp. 4273-4283, 2008. 